\title{
Ray-map migration of transmitted surface waves
}

\author{
Jing Li ${ }^{1}$ and Gerard T. Schuster ${ }^{1}$
}

\begin{abstract}
Near-surface normal faults can sometimes separate two distinct zones of velocity heterogeneity, where the medium on one side of the fault has a faster velocity than on the other side. Therefore, the slope of surface-wave arrivals in a common-shot gather should abruptly change near the surface projection of the fault. We present ray-map imaging method that migrates transmitted surface waves to the fault plane, and therefore it roughly estimates the orientation, depth, and location of the near-surface fault. The main benefits of this method are that it is computationally inexpensive and robust in the presence of noise.
\end{abstract}

\section{Introduction}

Geophysicists and engineers often require the accurate detection and location of geologic anomalies at shallow depths below the free surface. Unfortunately, the near-surface environment is often characterized by high noise levels, strong scattering, and significant spatial variations in rock properties. These impediments often prevent successful imaging of the nearsurface reflectivity or velocity by reflection-imaging methods. As an alternative, surface-wave imaging methods can be used to assess the subsurface geology for geotechnical applications (Socco et al., 2010; Dal Moro et al., 2015), crustal seismology (Campman et al., 2004; Shapiro et al., 2005), and fault identification associated with earthquake hazard assessment (Ivanov et al., 2006; Yu et al., 2014; AlTheyab et al., 2016). In addition, surface waves in the form of Stoneley waves are used to assess the rock properties around a well bore in the kilohertz range with sonic logs (Stevens and Day, 1986) as well as high-frequency vertical seismic profile data (Tang and Cheng, 1996) and crosswell data (Worthington, 1991).

The most common surface-wave imaging method is to invert the dispersion curves for either phase-velocity or S-velocity tomograms (Yamanaka and Ishida, 1996; Xia et al., 1999; Parolai et al., 2005; Dal Moro and Pipan, 2007; Lin et al., 2008; Li and Schuster, forthcoming). This inversion procedure is robust and can be used to assess the smoothly varying phase-velocity variations down to a depth of approximately $\lambda / 3$ (Stokoe et al., 1994; Park et al., 1999), where $\lambda$ is the longest effective wavelength associated with the surface-wave data.

One of the problems with inverting dispersion curves is that the resulting tomograms only give an estimate of the smoothly varying velocity in the subsurface. In these tomograms, it is difficult to identify sharp variations of velocity that might correspond to a fault. As an example, the S- and P-wave velocity tomograms in Figure $1 \mathrm{a}$ and $1 \mathrm{~b}$ suggest localized velocity anomalies but do not unambiguously delineate fault structures. In contrast, the rapid time shifts in the common-offset gather (COG) events in Figure 1c suggest the presence of nearsurface faults (or low-velocity zones), but their dip angles and depths are unknown. Therefore, subsurface images with much higher wavenumbers than Figure 1a and $1 \mathrm{~b}$ are required to reveal the depth and orientation of the faults.

To generate detailed images of fault zones, geophysicists proposed the migration of back-scattered surface waves (Snieder, 1986; Campman et al., 2004, 2005; Brandsberg-Dahl et al., 2007; Sinha et al., 2009; Hanafy et al., 2014; Sloan et al., 2015; AlTheyab et al., 2016). The migration procedure can be either a reverse time migration method (Almuhaidib and Toksöz, 2015), a Kirchhoff-like migration (Hyslop and Stewart, 2014; $\mathrm{Yu}$ et al., 2014), or a seismic scanning tunneling macroscope method (AlTheyab et al., 2016). However, back-scattered surface waves are not always easy to identify and extract from the records because their signal-to-noise ratio can be weak compared with the transmitted surface waves. Thus, imaging faults with noisy backscattered surface waves are often not possible.

To overcome the problem with weak backscattering, we propose ray-map migration of transmitted surface waves. This takes advantage of the fact that transmitted surface waves typically are an order-of-magnitude stronger than any other arrivals in the record, such as the much weaker reflections or backscattered sur-

\footnotetext{
${ }^{1}$ King Abdullah University of Science and Technology, Department of Earth Science and Engineering, Thuwal, Saudi Arabia. E-mail: jing.li@kaust .edu.sa; gerard.schuster@kaust.edu.sa.

Manuscript received by the Editor 25 January 2016; revised manuscript received 3 June 2016; published online 25 August 2016. This paper appears in Interpretation, Vol. 4, No. 4 (November 2016); p. SQ33-SQ40, 8 FIGS.

http://dx.doi.org/10.1190/INT-2016-0014.1. @ 2016 Society of Exploration Geophysicists and American Association of Petroleum Geologists. All rights reserved.
} 
face waves. The imaging condition for migrating transmitted surface waves is that there is an abrupt change in the slope of the transmitted surface waves because they cross a fault with a strong velocity contrast.

The abrupt slope change of surface-wave arrivals is most easily seen in the COGs in Figure 1c and illustrated in Figure 2 for the vertical fault-plane model.

Note the change in the slopes and arrival times at location $A$, as the surface waves propagate from one side of the dashed red box to the other side. In this example, the COG traces with midpoints $x>L$ only feel the influence of the $v_{2}$ medium, so the corresponding surface waves only propagate at that velocity. Here, $L$ is the half-offset between the source-receiver pair in the COG.

The crossover points (e.g., $A$ ) are delineated by the dark brown lines in Figure 2 and depend on the half-offset $L$ of the COG and the midpoint coordinate $x$ of the source-receiver pair in the COG. This crossover location is the midpoint at which the dashed black box, with width $2 L$, is just to the right of the fault, so a surface wave propagating from one side to the other only travels through the $v_{2}$ medium. The surface waves are only influenced by the media on both sides of the fault for source-receiver pairs with midpoints within the region of influence (ROI) in Figure 2, denoted by the brown triangular area between the crossover lines.

Traditional ray-map migration of reflections (Yilmaz, 2001) maps the reflection amplitude to the intersection
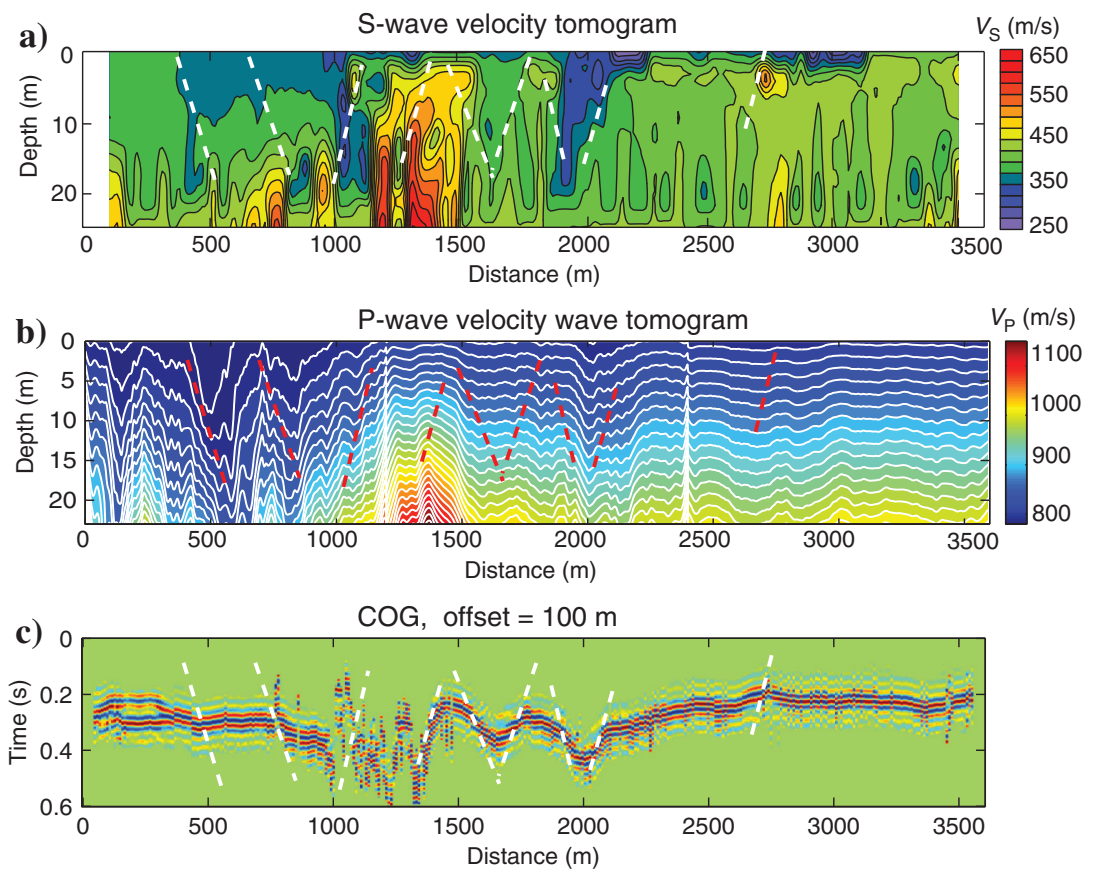

Figure 1. (a) The S-velocity tomogram obtained by inverting the dispersion curves in an East Africa data set, (b) P-velocity tomogram by wave-equation traveltime inversion of first arrivals, and (c) COGs at source-receiver offset of $100 \mathrm{~m}$ for data recorded in the East Africa survey. Dashed lines delineate sharp jumps in the velocity and the traveltimes seen, respectively, in the S-velocity tomogram and the COG. point $(x, z)$ between the source's direct rays and the reflection rays. For surface waves, we map the amplitude of the early arrival surface wave recorded at the crossover trace to the deepest portion $(x-L, \lambda / 3)$ of the fault, in which the surface wave could be significantly influenced. Equivalently, the crossover events at $A$ are mapped to their place of origin, namely, the lower left corner of the dashed red box at $(x-L, \lambda / 3)$ that intersects the fault in Figure 2. We choose the depth $z=\lambda / 3$ because surface waves are primarily influenced by the medium properties to a depth of $\lambda / 3$ (Stokoe et al., 1994; Park et al., 1999). Consequently, events at lower frequencies map to deeper portions of the fault as illustrated in the bottom illustration of Figure 2. The imaging condition is that the surface-wave arrivals at crossover oints are migrated to the depth $\lambda / 3$ along the fault with a strong velocity contrast. Migrating transed surface waves is similar to migrating transmitted arrivals to their conversion points (Sheley and alor, 2003) along the interface of a strong velocity role of the migrated crossover point.

The next section describes the algorithmic details for ray-map migration of surface waves in the COG domain. A workflow is described, and its effectiveness is then illustrated with numerical examples. Synthetic data tests and field data examples show the benefits and limitations of ray-map migration of surface waves. The last section presents our conclusions.

\section{Ray-map migration of transmitted surface waves}

We now present the algorithm for migrating surface waves to the fault boundary delineated by the dashed black lines in Figure 2. For convenience, the left side of the vertical fault has the homogeneous velocity $v_{1}=1 / s_{1}$ and the right side has the velocity $v_{2}=$ $1 / s_{2}$. The COG traveltime $t(x)$ for a narrow-band surface wave with the COG midpoint at $x$ is given as

$$
t(x)=\left\{\begin{array}{cc}
2 L s_{1} & -L \geq x \\
(L-x) s_{1}+(L+x) s_{2} & L \geq x \geq-L \\
2 L s_{2} & x \geq L
\end{array}\right\},
$$

where $2 L$ is the offset between the source and receiver, $L$ is the half-offset at the midpoint location $x, s_{1}$ is the slowness to the left of the fault, and $s_{2}$ is the slowness to the right of the fault. Here, it is assumed that the traveltime $t(x)$ has been time shifted to account for a constant time delay. As illustrated in Figure 2, equation 1 says that the slope 
of the traveltime curve $t(x)$ should be zero for $x<-L$ and $x \geq L$, otherwise the slope is linearly proportional to the midpoint coordinate. The proportionality constant is the slowness change $s_{2}-s_{1}$ across the fault.

\section{Traveltimes and a dipping-fault model}

The traveltime formula for the COG trace associated with the dipping fault in Figure 3 is now derived in this section. Here, we assume a narrow-band arrival that propagates with a weighted average S-velocity to the depth of $\lambda / 3$. The average slowness $\bar{s}(x)$ below the midpoint $x$ is approximated by

$$
\bar{s}(x)=\left\{\begin{array}{cc}
s_{1} & 0 \geq x \\
s_{2} \frac{x}{\frac{1}{3} \tan \phi}+s_{1}\left(1-\frac{x}{\frac{\lambda}{3} \tan \phi}\right) & \frac{\lambda}{3} \tan \phi \geq x \geq 0 \\
s_{2} & x \geq \frac{\lambda}{3} \tan \phi
\end{array}\right\},
$$

where average slowness $\bar{s}(x)$ is only a rough approximation to the phase velocity at the specified frequency, but it is sufficient for the rough estimation of the fault's dip angle by ray-map migration.

a) COG in time-midpoint-offset volume

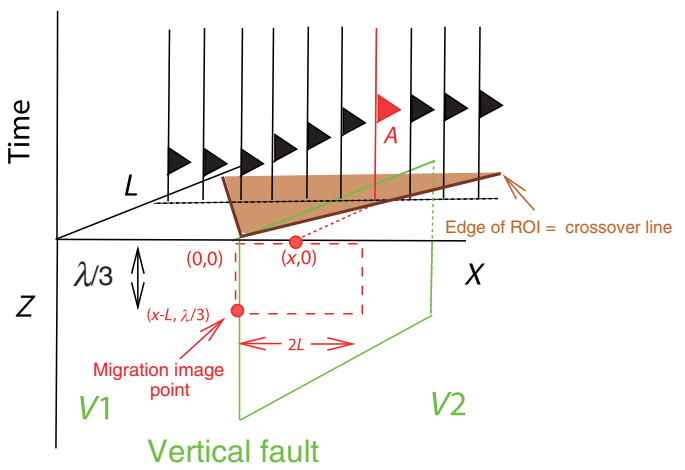

b) Lower frequencies map to deeper points

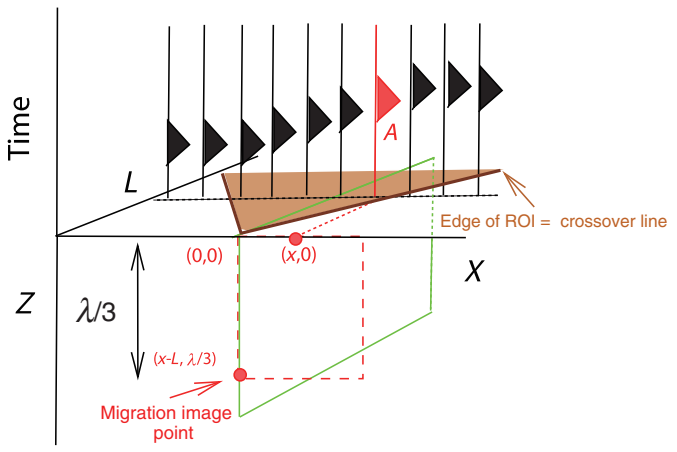

Figure 2. (a) The COG in a time-midpoint-offset volume, where the midpoint coordinate is $x$ and the half-offset coordinate is $L$. The crossover arrival (i.e., the red event at A) is mapped to the migration image point at $(x-L, \lambda / 3)$; only crossover events along the edge of the brown ROI are migrated to their points of origin along the fault. (b) Same as top illustration except the data have been low-pass filtered, so that the dashed red box and the image point are deeper.
The traveltime $t(x)$ of the narrow-band surface wave for the dipping-fault model in Figure 3 with midpoint coordinate $x$ is therefore given by

$$
\begin{aligned}
- & L<x<L ; t(x) \\
& =\int_{x-L}^{x+L} \bar{s}\left(x^{\prime}\right) d x^{\prime} \\
& =(L-x) s_{1}+\int_{0}^{M} \bar{s}\left(x^{\prime}\right) d x^{\prime}+\int_{M}^{x+L} s_{2} d x^{\prime} \\
& =(L-x) s_{1}+\left.\frac{s_{2}-s_{1}}{2 \lambda / 3 \tan \phi} x^{2}\right|_{0} ^{M}+\left.s_{1} x\right|_{0} ^{M}+\left.s_{2} x\right|_{M} ^{x+L},
\end{aligned}
$$

where $M=\min (\lambda / 3 \tan \phi, x+L)$. In the limit of small $\phi$, the above formula reduces to

$$
\begin{aligned}
-L<x<L ; t(x) & =(L-x) s_{1}+\left.s_{2} x\right|_{0} ^{x+L} \\
& =(L-x) s_{1}+(L+x) s_{2},
\end{aligned}
$$

which agrees with equation 1 for a vertical fault.

Case 1: $M=\lambda / 3 \tan \phi$ : For a sufficiently small dip angle $\phi$ and large enough $x+L$, such that $M=$ $\min (\lambda / 3 \tan \phi, x+L)=\lambda / 3 \tan \phi$, equation 3 becomes

$$
\begin{aligned}
t(x)= & (L-x) s_{1}+\frac{s_{2}-s_{1}}{6} \lambda \tan \phi+s_{1} \lambda / 3 \tan \phi \\
& +\left.s_{2} x\right|_{\lambda / 3 \tan \phi} ^{x+L} \\
= & (L-x) s_{1}+(x+L) s_{2}-\lambda\left[\frac{s_{2}-s_{1}}{6}\right] \tan \phi .
\end{aligned}
$$

Thus, $t(x)$ is a linear function of the midpoint coordinate, and its slope is equal to $s_{2}-s_{1}$. Knowing $s_{1}$ and $s_{2}$ from the data, the value of $\tan \phi$ can be determined by plotting values of $t(x)$ against the midpoint coordinate $x$ and finding the intercept value $t(0)$ to give $\phi=\tan ^{-1}\left\{\left(6\left[t(0)-L\left(s_{1}+s_{2}\right)\right]\right) / \lambda\left(s_{1}-s_{2}\right)\right\}$.

A more practical means to determine dip angle is to define the ROI, as the area in the $x-L$ domain where the slopes of surface-wave events are nonzero. The edge of the ROI is where the slope abruptly changes, i.e., the crossover points denoted by the dark brown lines in Figure 2 or 4 . For the vertical fault in Figure 2,

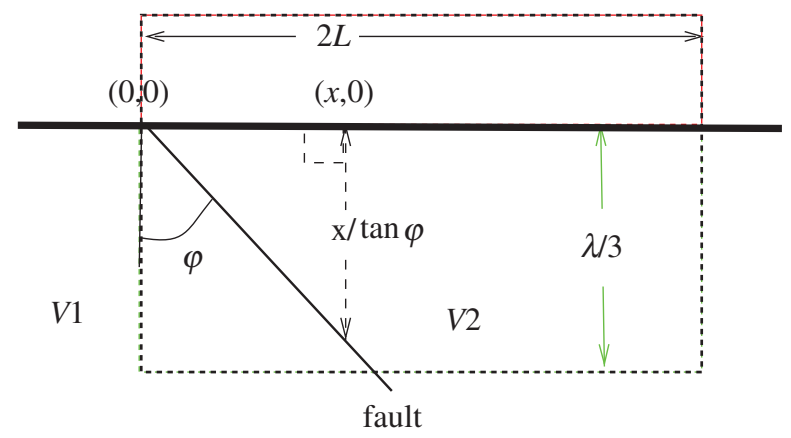

Figure 3. Dipping-fault model in which the surface wave is not sensitive to velocities deeper than $\lambda / 3$ and the COG midpoint has the coordinate value $x$. The offset value of the COG trace is $2 L$. 
the ROI is in the shape of a symmetrical triangle, with the vertical symmetry plane at the fault. In this case, the amplitude of the surface-wave event $A$ is mapped to the fault at the image point $(x-L, \lambda / 3)$. To account for a finite-wavelength resolution, the wavelet can be laterally smeared onto a wavelength-sized area around this image point.

In contrast, if the fault is dipping as shown in Figure 4, the ROI is asymmetrical. If the wavelength is selected, such that $x+L>\tan \phi \lambda / 3$, then the crossover events along the edge of the ROIs in Figure 4 are mapped to the fault at $(x-L, \lambda / 3)$. The dip angle of the fault can be estimated from the formula $\phi=\tan ^{-1}$

\section{ROls for dipping fault model}
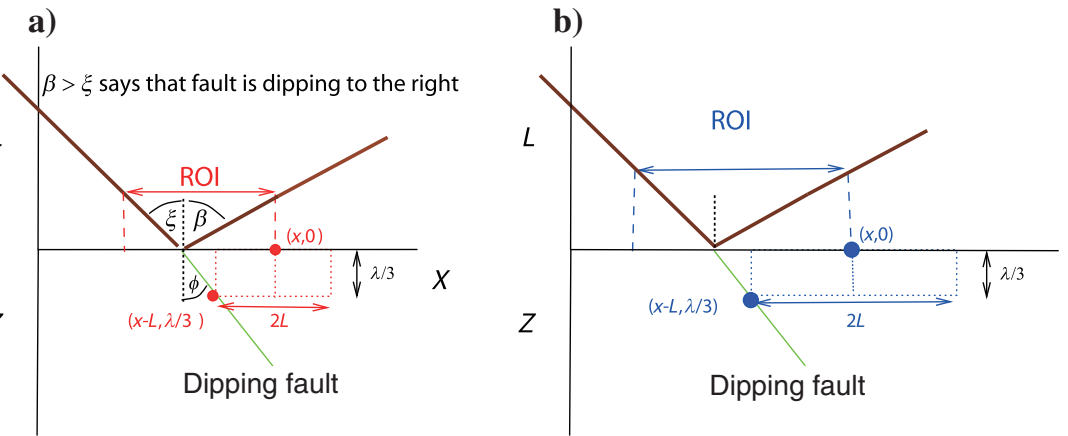

Figure 4. Dipping-fault model and ROIs at two different offset values of $L$ and a fixed wavelength $\lambda$. Unlike the vertical fault, the ROI for a dipping fault does not form a symmetrical region in $x-L$ space. The fault is dipping to the right, if $\beta>\xi$, where the angles $\beta$ and $\xi$ of the brown crossover lines are measured to the vertical.
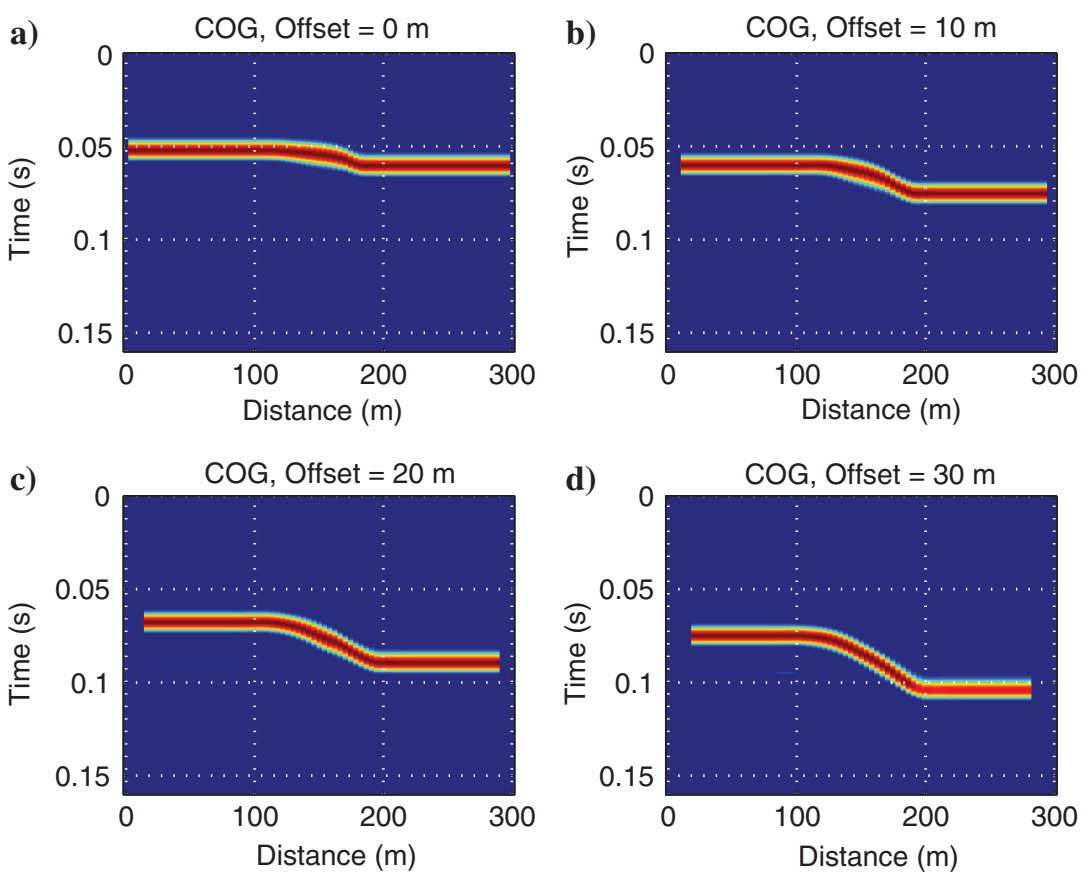

Figure 5. The COGs for different values of offset $L$ in a dipping-fault model. As expected, the ROI increases in width as $L$ becomes larger. The nonzero amplitudes of the surface-wave events are denoted as reddish colors.
$\left[3\left(x_{c}-L\right) / \lambda\right]$, where $x_{c}$ is the crossover point for the pecified value of $L$.

Case 2: $\quad M=x+L$ : For a sufficiently large avelength $\lambda$, such that $M=\min (\lambda / 3 \tan \phi, x+L)=$ , equation 3 becomes

$$
\begin{aligned}
t(x) & =(L-x) s_{1}+\left.\frac{s_{2}-s_{1}}{2 \lambda / 3 \tan \phi} x^{2}\right|_{0} ^{x+L}+\left.s_{1} x\right|_{0} ^{x+L} \\
& =2 L s_{1}+\frac{s_{2}-s_{1}}{2 \lambda / 3 \tan \phi}(x+L)^{2},
\end{aligned}
$$

which is a quadratic function of the midpoint coordinate $x$ and offset value $L$. In theory, the value of $\tan \phi$ can be computed by determining the curvature $\left[d^{2} t(x)\right] / d x^{2}$ of the traveltime curve, if $s_{1}$ and $s_{2}$ are known. With the two cases, we will introduce the basic workflow for ray-map migration.

\section{Workflow for ray-map migration}

The following ray-map procedure is proposed for imaging near-surface faults, as long as the average velocity on one side of the fault is significantly different than on the other side:

1) Bandpass the COG data into $N$ narrow frequency bands, so that the $i$ th bandpassed COG has a dominant frequency $f_{i}$ related to the dominant wavelength $\lambda_{i}=c_{i} / f_{i}$.

2) Pick the early arrival surface waves at the crossover points $\mathbf{A}=\left(x_{A}, 0\right)$ in Figure 2 for each value of the offset $L$. Map the arrival to the fault plane at $\left(x_{A}-L, z_{i}\right)=\left(x_{A}-L, \lambda_{i} / 3\right)$, i.e.

$$
\begin{aligned}
& m\left(x-L, z_{i}, L\right) \\
& \quad=\cos \left(\pi\left(x_{A}-x\right) / \lambda_{i}\right) \\
& \text { for } z_{i}=\lambda_{i} / 3 \text { and } \\
& x_{A}-\lambda / 2 \leq x \leq x_{A}+\lambda / 2 .
\end{aligned}
$$

The width of this image is one wavelength and centered at $\left(x_{A}-L, \lambda_{i} / 3\right)$, where the phase velocity $c_{i}$ is estimated from the data. The offset value $L$ should meet the condition $\lambda / 3 \tan \phi<x_{A}+L$ at the crossover point $x_{A}$.

3) Repeat step (3) for $i=[1,2,3, \ldots, N]$ to form the final-migration image:

$$
M\left(x, z_{i}\right)=\sum_{L} m\left(x, z_{i}, L\right),
$$

where the summation is over a small range of offset values $L$ that satisfy the condition $|L-x|>\lambda_{i} / 3$ at the 
crossover midpoint value $x$. As a sanity test, the migration images at different offset values $L$ are compared for their similarity. This is similar to checking for flat events in a common image gather (CIG), except the CIG for the migrated surface waves can be used to correct for incorrect estimates of fault dip and location. Here, care must be taken to properly choose the offset value $2 L$ of the COG. If it is too large, then the spatial resolution of the migration image becomes worse as many geologic features can influence the first arrival. If $2 L$ is too small, then the surface wave might not have a chance to develop, and there is more uncertainty in estimating the values of the slope in the ROI. Hence, we recommend $L>2 \lambda$.

4) An alternative to the above procedure is to simply map the traveltimes $t\left(x, L, \lambda_{i}\right)$ of the early arrival surface waves to depth using

$$
m\left(x-L, z=\lambda_{i} / 3\right)=t\left(x, L, \lambda_{i}\right)
$$

The fault plane is located by identifying the strong change in traveltimes in $m\left(x-L, z=\lambda_{i} / 3\right)$. Another possibility is to apply a Laplacian operation to $m\left(x-L, z=\lambda_{i} / 3\right)$ followed by a smoothing filter, or by applying $d / d x$ and smoothing to $t\left(x, L, \lambda_{i}\right)$ and migrating the result.

The above procedure is denoted as ray-map migration because it maps the surface-wave arrival at the crossover point to the average depth at which that arrival is sensitive to S-velocity variations.
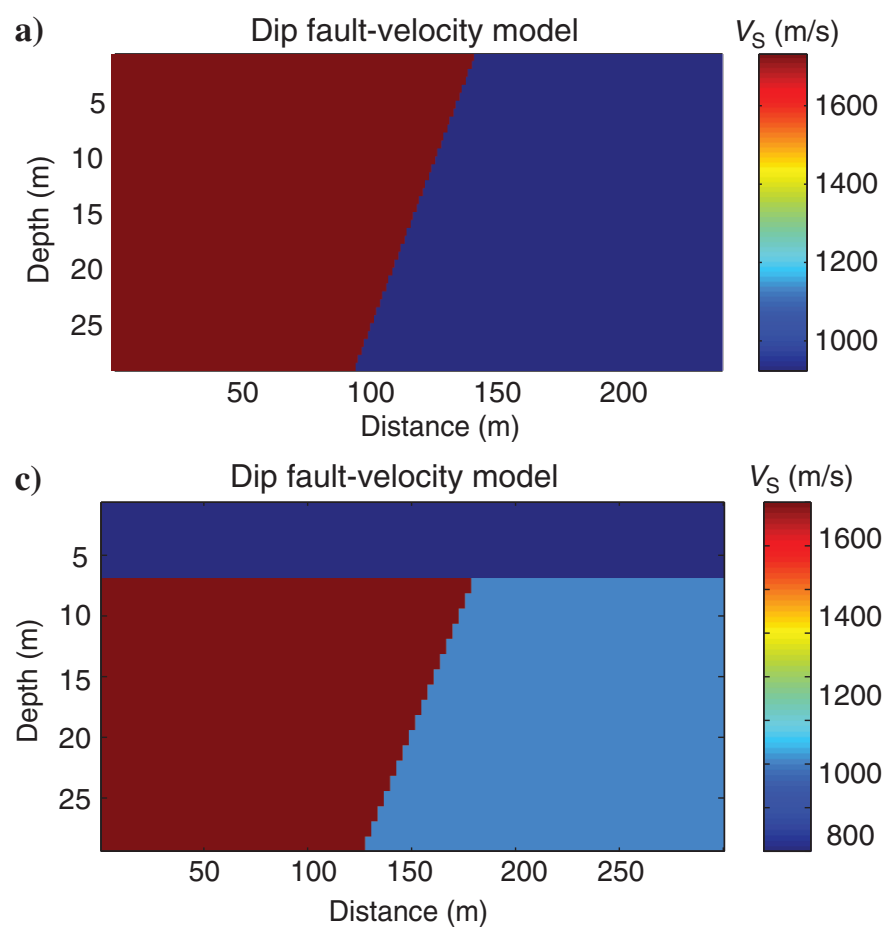

\section{Numerical results}

Solutions to the $2 \mathrm{D}$ elastic-wave equation are computed for a dipping-fault model. The windowed COGs are generated from the synthetic shot gathers and are shown in Figure 5a-5d. The traveltimes of the COGs increase with increasing values of $L$ and the slope varies linearly with $x$ in the ROI, just as predicted in equation 5 .

\section{Synthetic data}

Finite-difference solutions to the 2D elastic-wave equation are used to generate the COGs for the Figure $6 \mathrm{a}$ and $6 \mathrm{c}$ models. We use equation 9 to migrate the COGs, so that all early arrival surface waves in the COGs are mapped to depth, including noncrossover events (Figure 6b and 6d). In both cases, the "migration images" roughly estimate the location of the fault planes by identifying the interfaces (dashed black lines in Figures $6 \mathrm{~b}$ and $6 \mathrm{~d}$ ) that define sharp jumps in color.

\section{KAUST data}

Seismic data were collected next to the KAUST campus along a $1.2 \mathrm{~km}$ line. The COG with an offset value of $2 L=20 \mathrm{~m}$ is shown in Figure $7 \mathrm{a}$, and the associated migration image is shown in Figure 7b. As in the synthetic examples, equation 9 is used to map the traveltimes of the early arrival surface waves in the COGs regardless if the events are at a crossover point. As a consistency check, the COG with $2 L=50 \mathrm{~m}$ is also migrated to give the image in Figure 7c.

The migration images in Figure $7 b$ and $7 c$ with offset values $2 L=20$ and $50 \mathrm{~m}$ show a steeply dipping fault
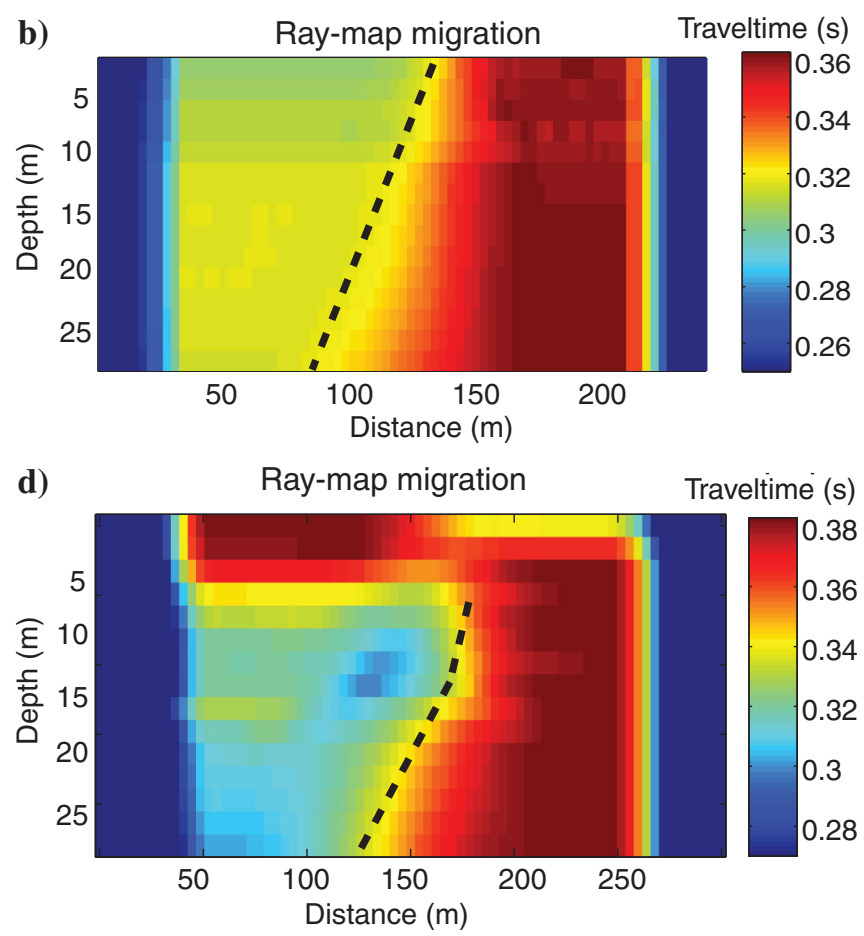

Figure 6. (a) Simple- and (c) layered-fault models, with S-velocity "migration images" to the right. Hotter colors in the migration images correspond to longer traveltimes and suggest low-velocity sediments on the upthrown side of the dashed normal fault. 
(dashed white lines) that is consistent from one COG to the other. This is the expected location of the Qademah fault based on evidence from other geophysical methods (Hanafy et al., 2015; Li and Hanafy, 2016).
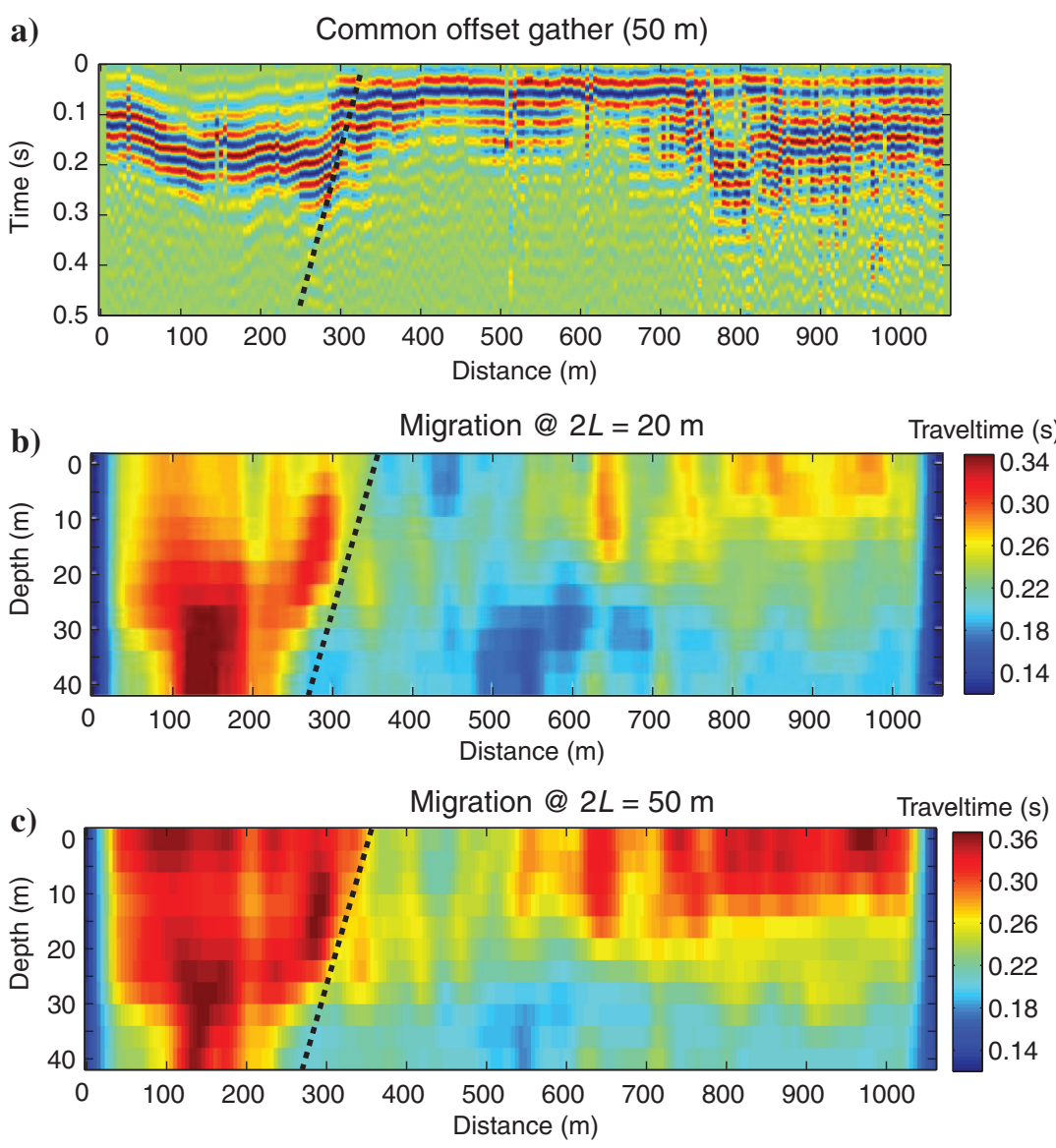

Figure 7. (a) The KAUST COG and migration images computed from COGs with offsets of (b) $2 L=20$ and (c) $50 \mathrm{~m}$. Hotter colors in the migration images correspond to longer traveltimes and suggest low-velocity sediments on the downthrown side of the dashed normal fault.
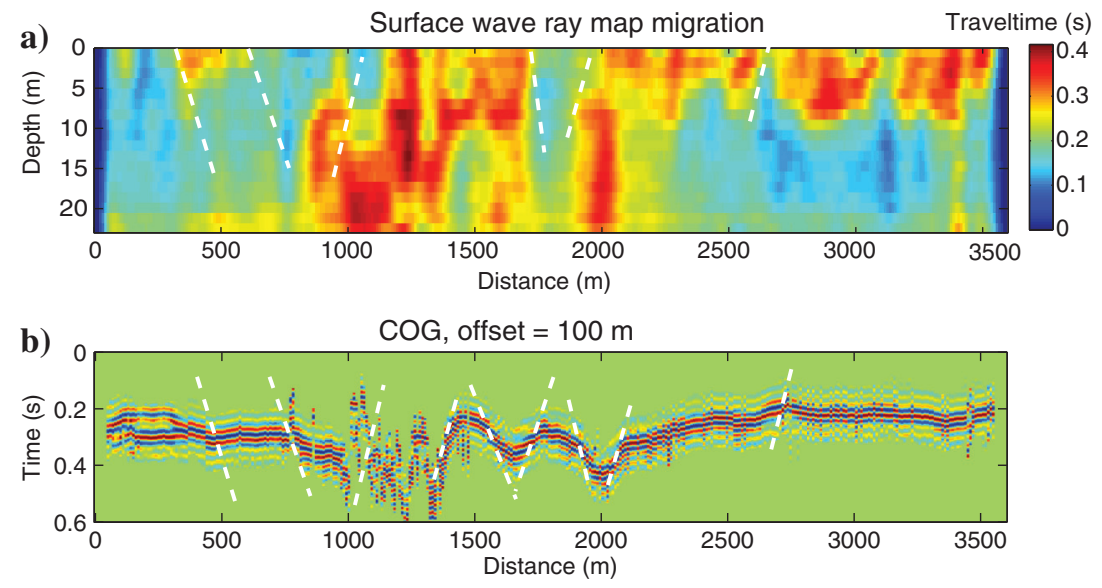

Figure 8. (a) Migration image and (b) COG estimated from seismic data collected in East Africa. The dashed white lines were drawn only if the migration image suggested a fault-like structure.

\section{ast Africa data}

Seismic profiles were recorded over a $3.2 \mathrm{~km}$ long line in East Africa, and the COG at $2 L=100 \mathrm{~m}$ is shown in Figure 8b. The COGs were band-pass filtered, and the resulting first-arrival surface-wave traveltimes were used in equation 5 to determine the migration image in Figure 8a. The interpreted faults are denoted by dashed white lines and compare well with those shown in the Figure 1a tomogram inverted from the dispersion curves. This basin is under extension, so most of the faults are normal faults, as indicated by the low velocities in the graben zones.

\section{Summary and discussion}

An efficient algorithm for ray-map migration of transmitted surface waves is presented for imaging a near-surface fault boundary. The assumptions are (1) the simple fault model is characterized by a strong velocity contrast across the fault plane, (2) the crossover arrivals should be mapped to the bottom left corner of the dashed black boxes in Figure 1 for a fault dipping to the right, and (3) here, the thickness of the box is crudely estimated to be $\lambda / 3$. If the ROI is asymmetrical and tilts to the right, then the fault is dipping to the right. Otherwise, it is a left-dipping fault. The crossover line is picked in the COG profile, so that ray-map migration avoids the complexity and expense of surface-wave inversion methods. Ray-map migration should robustly provide a qualitative model of the fault geometry, even in the presence of strong noise.

The main problem with ray-map migration of surface waves is that it only provides a rough approximation to the actual dip and location of the fault plane. However, combining the interpretation of the velocity tomogram with the ray-map migration image can significantly reduce the uncertainty in identifying the location and dip angle of hidden faults.

\section{Acknowledgments}

We thank the 2016 sponsors of Center for Subsurface Imaging and Fluid Modeling (CSIM) at King Abdullah University of Science and Technology (KAUST) for their support. 


\section{References}

Almuhaidib, A. M., and M. N. Toksöz, 2015, Imaging of near-surface heterogeneities by scattered elastic waves: Geophysics, 80, no. 4, A83-A88, doi: 10.1190/ geo2014-0416.1.

AlTheyab, A., F.-C. Lin, and G. T. Schuster, 2016, Imaging near-surface heterogeneities by natural migration of backscattered surface waves: Geophysical Journal International, 204, 1332-1341, doi: 10.1093/gji/ggv511.

Brandsberg-Dahl, S., B. Hornby, and X. Xiao, 2007, Migration of surface seismic data with VSP Green's functions: The Leading Edge, 26, 778-780, doi: 10.1190/1.2748496.

Campman, X., K. Van Wijk, C. Riyanti, J. Scales, and G. Herman, 2004, Imaging scattered seismic surface waves: Near Surface Geophysics, 2, 223-230, doi: 10.3997/ 1873-0604.2004019.

Campman, X. H., K. van Wijk, J. A. Scales, and G. C. Herman, 2005, Imaging and suppressing near-receiver scattered surface waves: Geophysics, 70, no. 2, V21-V29, doi: 10.1190/1.1884831.

Dal Moro, G., R. M. M. Moura, and S. S. Moustafa, 2015, Multi-component joint analysis of surface waves: Journal of Applied Geophysics, 119, 128-138, doi: 10.1016/j. jappgeo.2015.05.014.

Dal Moro, G., and M. Pipan, 2007, Joint inversion of surface wave dispersion curves and reflection travel times via multi-objective evolutionary algorithms: Journal of Applied Geophysics, 61, 56-81, doi: 10.1016/j.jappgeo.2006 .04 .001 .

Hanafy, S. M., A. AlTheyab, and G. T. Schuster, 2015, Controlled noise seismology: 85th Annual International Meeting, SEG, Expanded Abstracts, 5102-5106.

Hanafy, S. M., G. T. Schuster, and B. Danielsen, 2014, Fault detection by surface seismic scanning tunneling macroscope: Field test: 84th Annual International Meeting, SEG, Expanded Abstracts, 4608-4612.

Hyslop, C., and R. Stewart, 2014, Mapping and migrating reflected surface waves: Near Surface Geoscience 2014 - 20th European Meeting of Environmental and Engineering Geophysics, 28-32.

Ivanov, J., R. D. Miller, P. Lacombe, C. D. Johnson, and J. W. Lane Jr., 2006, Delineating a shallow fault zone and dipping bedrock strata using multichannel analysis of surface waves with a land streamer: Geophysics, 71, no. 5, A39-A42, doi: 10.1190/1.2227521.

Li, J., and S. Hanafy, 2016, Skeletonized inversion of surface wave: Active source vs controlled noise comparison: Interpretation, 4, SH11-SH19, doi: 10.1190/int-2015-0174.1.

Li, J., and G. T. Schuster, forthcoming, Skeletonized waveequation surface wave dispersion inversion: Geophysical Journal International.

Lin, F.-C., M. P. Moschetti, and M. H. Ritzwoller, 2008, Surface wave tomography of the western United States from ambient seismic noise: Rayleigh and Love wave phase velocity maps: Geophysical Journal International, 173, 281-298, doi: 10.1111/j.1365-246X.2008.03720.x.
Park, C. B., R. D. Miller, and J. Xia, 1999, Multichannel analysis of surface waves: Geophysics, 64, 800-808, doi: 10.1190/1.1444590.

Parolai, S., M. Picozzi, S. Richwalski, and C. Milkereit, 2005, Joint inversion of phase velocity dispersion and $\mathrm{h} / \mathrm{v}$ ratio curves from seismic noise recordings using a genetic algorithm, considering higher modes: Geophysical research letters, 32, 308-312, doi: 10.1029/2004GL021115.

Shapiro, N. M., M. Campillo, L. Stehly, and M. H. Ritzwoller, 2005, High-resolution surface-wave tomography from ambient seismic noise: Science, 307, 1615-1618, doi: 10.1126/science.1108339.

Sheley, D., and G. T. Schuster, 2003, Reduced-time migration of transmitted PS waves: Geophysics, 68, 16951707, doi: 10.1190/1.1620643.

Sinha, S., B. Hornby, and R. Ramkhelawan, 2009, 3D depth imaging of surface seismic using VSP measured Green's function: 79th Annual International Meeting, SEG, Expanded Abstracts, 4125-4128.

Sloan, S. D., S. L. Peterie, R. D. Miller, J. Ivanov, J. T. Schwenk, and J. R. McKenna, 2015, Detecting clandestine tunnels using near-surface seismic techniques: Geophysics, 80, no. 5, EN127-EN135, doi: 10.1190/geo2014-0529.1.

Snieder, R., 1986, 3D linearized scattering of surface waves and a formalism for surface wave holography: Geophysical Journal International, 84, 581-605, doi: 10 .1111/j.1365-246X.1986.tb04372.x.

Socco, L. V., S. Foti, and D. Boiero, 2010, Surface-wave analysis for building near-surface velocity models established approaches and new perspectives: Geophysics, 75, no. 5, 75A83-75A102, doi: 10.1190/1.3479491.

Stevens, J. L., and S. M. Day, 1986, Shear velocity logging in slow formations using the Stoneley wave: Geophysics, 51, 137-147, doi: 10.1190/1.1442027.

Stokoe, K. H., S. G. Wright, J. A. Bay, and J. M. Roesset, 1994, Characterization of geotechnical sites by SASW method, Geophysical characterization of sites: Oxford and IBH Publication Company, 15-25.

Tang, X., and C. Cheng, 1996, Fast inversion of formation permeability from Stoneley wave logs using a simplified Biot-Rosenbaum model: Geophysics, 61, 639-645, doi: 10.1190/1.1443993.

Worthington, M., 1991, Cross-well continuity logging using Stoneley waves at the Whitchester borehole test site: 53rd EAEG Meeting, 180-181.

Xia, J., R. D. Miller, and C. B. Park, 1999, Estimation of nearsurface shear-wave velocity by inversion of Rayleigh wave: Geophysics, 64, 691-700, doi: 10.1190/1.1444578.

Yamanaka, H., and H. Ishida, 1996, Application of genetic algorithms to an inversion of surface-wave dispersion data: Bulletin of the Seismological Society of America, 86, 436-444.

Yilmaz, Ö., 2001, Seismic data analysis: SEG.

Yu, H., B. Guo, S. Hanafy, F.-C. Lin, and G. T. Schuster, 2014, Direct detection of near-surface faults by migration of back-scattered surface waves: 84th Annual 
International Meeting, SEG, Expanded Abstracts, 2135-2139.

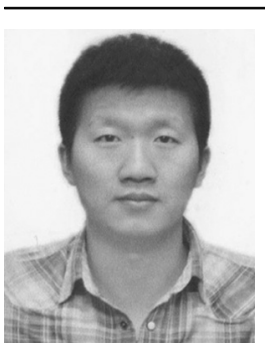

Jing Li received a master's degree (2011) and a Ph.D. (2014) in applied geophysics from Jilin University, China. He is a postdoc fellow at CSIM group, KAUST, where he focuses on seismic surface wave inversion and data processing. He worked as a visiting researcher at Delaware State University, USA, from 2012 to 2014 . His work focused on the advance math method in geophysics application. His current research interests include seismic interferometry for subsurface monitoring and GPR simulation and inversion for shallow subsurface, through-wall detection and lunar radar detection.

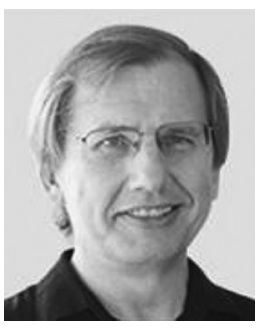

Gerard T. Schuster received an M.S. (1982) and a Ph.D. (1984) from Columbia University and was a postdoctoral researcher there from 1984 to 1985. From 1985 to 2009 , he was a professor of geophysics at the University of Utah. He left Utah to start his current position as professor of geophysics at KAUST in 2009. He is currently a professor of geophysics at KAUST and an adjunct professor of geophysics at the University of Utah. He was the founder and director of the Utah Tomography and Modeling/Migration consortium from 1987 to 2009, and he is now the codirector and founder of the Center for Fluid Modeling and Seismic Imaging at KAUST. He has helped pioneer seismic interferometry and its practical applications in applied geophysics through his active research program and through his extensive publications, including his book Seismic interferometry (Cambridge Press, 2009). He also has extensive experience in developing innovative migration and inversion methods for exploration and earthquake seismology. He received several teaching and research awards while at the University of Utah. He was the editor of Geophysics from 2004 to 2005, and he was awarded SEG's Virgil Kauffman gold medal in 2010 for his work in seismic interferometry. 